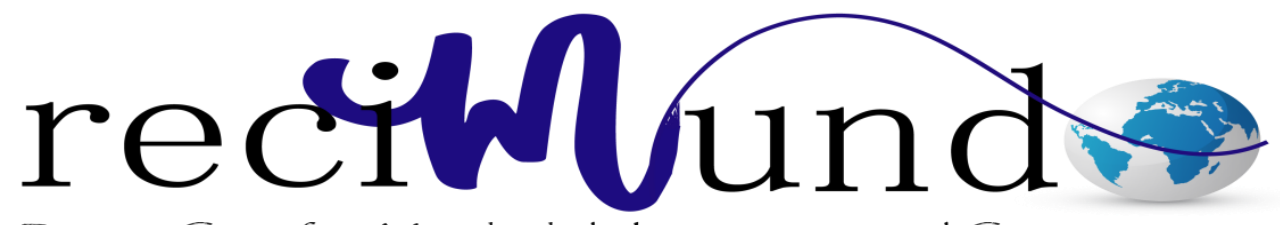

Revista Científica Mundo de la Investigación y el Conocimiento

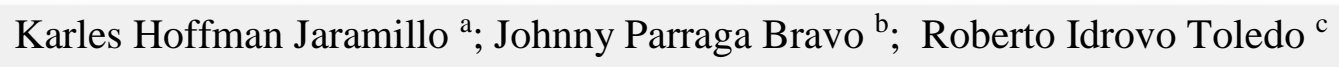

Evaluación ergonómica de los puestos de trabajo del área operativa de siembra y empaque

Revista Científica Mundo de la Investigación y el Conocimiento. Vol. 1 núm., 5, diciembre, 2017, pp. 41-51

DOI: 10.26820/recimundo/1.5.2017.41-51

Editorial Saberes del Conocimiento 


\section{Evaluación ergonómica de los puestos de trabajo del área operativa de siembra y empaque}

Vol. 1, núm. 5., (2017)

Karles Hoffman Jaramillo; Johnny Parraga Bravo; Roberto Idrovo Toledo

\section{RESUMEN}

Con el propósito de mejorar las condiciones laborales de los trabajadores, la productividad y calidad de sus servicios, la empresa agroindustrial procesadora de piñas Agroeden, decidió efectuar una evaluación ergonómica de los puestos de trabajo del área operativa para gestionar los riesgos laborales detectados. Con este fin, fue necesario analizar los niveles de riesgos laboral ergonómicos de forma cualitativa y cuantitativa en la entidad, el mismo que fue aplicado a 2 procesos de trabajo del área Operativa que hacen referencia a siembra y empaque, en el cual se valoró los puestos de trabajo, posturas forzadas y el confort ergonómico emitiendo propuestas de mejora para el ambiente laboral. Al efectuar la identificación de peligros y evaluación de riesgo laborales de la organización esta se encuentra en nivel "Moderado". Al analizar la sintomatología osteomuscular, el 10\% de los trabajadores señala sentir molestias debido a hábitos posturales y esfuerzos derivados a la actividad que realiza durante la jornada laboral. La evaluación proporciono información para elaborar una Matriz cualitativa de Riesgo Laborales, estableciendo que los puestos de trabajo que exponen mayor nivel riesgo laboral son: siembra y empaque por posturas forzadas y repetitividad de la tarea.

Palabras clave: Salud; laboral; trabajadores; ergonomía; trastornos. 


\title{
Evaluación ergonómica de los puestos de trabajo del área operativa de siembra y empaque
}

Vol. 1, núm. 5., (2017)

Karles Hoffman Jaramillo; Johnny Parraga Bravo; Roberto Idrovo Toledo

\begin{abstract}
With the purpose of improving the working conditions of the workers, the productivity and quality of their services, Agroeden pineapple agroindustrial processing company decided to carry out an ergonomic evaluation of the work areas of the operational area to manage the detected work risks. To this end, it was necessary to analyze the levels of ergonomic occupational risks in a qualitative and quantitative manner in the entity, which was applied to 2 work processes in the Operative area that refer to sowing and packaging, in which the positions were assessed of work, forced postures and ergonomic comfort by issuing proposals for improvement for the work environment. When carrying out the identification of hazards and evaluation of occupational risk of the organization, it is at the "Moderate" level. When analyzing musculoskeletal symptoms, $10 \%$ of workers report feeling discomfort due to postural habits and efforts derived from the activity performed during the workday. The evaluation provided information to prepare a qualitative Occupational Risk Matrix, establishing that the jobs that expose the highest level of occupational risk are: sowing and packing by forced postures and repetitiveness of the task.
\end{abstract}

Keywords: Health; labor; workers; ergonomics; disorders. 


\section{Evaluación ergonómica de los puestos de trabajo del área operativa de siembra y empaque}

Vol. 1, núm. 5., (2017)

Karles Hoffman Jaramillo; Johnny Parraga Bravo; Roberto Idrovo Toledo

\section{Introducción.}

Históricamente el trabajo ha representado a las sociedades en sí mismas, pues es el hecho que distingue al ser humano en su quehacer, y le otorga al colectivo una caracterización propia. Así, con el devenir de los tiempos, hombres y mujeres han estado sometidos a situaciones referidas al trabajo, las cuales implican, inevitablemente, la inminencia de una situación de riesgo para su salud o su vida. (Rodríguez, 2009)

Según la organización mundial de la salud se produce en el mundo, únicamente en la industria unos 50 millones de accidentes cada año, es decir una media de 160.000 al día. Por ellos se estima mueren aproximadamente 100.000 personas por año y muy probablemente estas cifras estén por debajo de la realidad. Cada año 1.500.000 trabajadores quedan inválidos para el resto de su vida. A estas cifras se debe añadir los millones de trabajadores víctimas de enfermedades contraídas en los lugares de trabajo. Se debería asimismo agregar, si se conociera su cifra, los accidentes graves y numerosos que se producen en la agricultura, los trabajos forestales y las plantaciones. En los países industrializados en promedio anual, uno de cada 10 trabajadores de la industria sufre heridas en el trabajo y se estima que en algunas ramas de actividad esa relación es en todo el mundo de uno cada 3. Se trata de tragedias humanas que podrían evitarse, pero que ocurren en el mundo cada día. (Polanco Trochez, Rodríguez Mosquera, \& Vidal Mosquera, 2011) 


\section{Evaluación ergonómica de los puestos de trabajo del área operativa de siembra y empaque}

Vol. 1, núm. 5., (2017)

Karles Hoffman Jaramillo; Johnny Parraga Bravo; Roberto Idrovo Toledo

\section{Imagen $N^{\circ}$ 1: Esquema de un sistema de gestión preventiva en base al plan de prevención.}

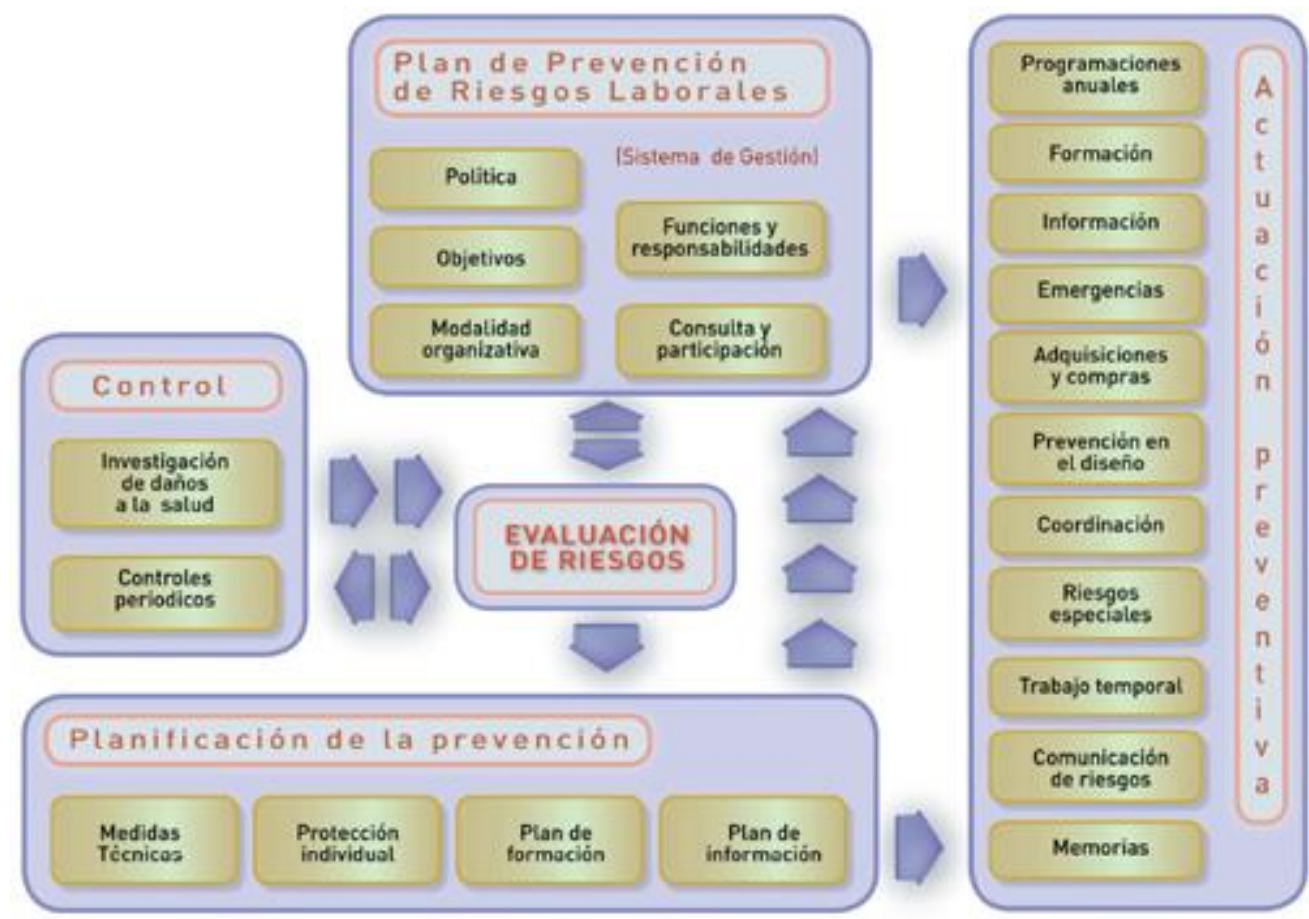

Fuente: (Salinas Garrido, 2008)

Las condiciones sociales y materiales en que se realiza el trabajo pueden afectar el estado de bienestar de las personas en forma negativa. Los daños a la salud más evidentes y visibles son los accidentes del trabajo. De igual importancia son las enfermedades profesionales, aunque se sepa menos de ellas. Los daños a la salud por efecto del trabajo resultan de la combinación de diversos factores y mecanismos. (Parra, 2003)

La evaluación de riesgos laborales constituye la base fundamental para la prevención de accidentes y enfermedades de origen laboral dentro de las organizaciones. Pero adicionalmente también se debe considerar que las medidas preventivas sirven para garantizar la productividad y calidad sostenible de los procesos y servicios que ofrecen las empresas. Una de las 


\section{Evaluación ergonómica de los puestos de trabajo del área operativa de siembra y empaque}

Vol. 1, núm. 5., (2017)

Karles Hoffman Jaramillo; Johnny Parraga Bravo; Roberto Idrovo Toledo

principales barreras al momento de mejorar el medio ambiente laboral, es la poca inversión e interés de los empresarios en temas de seguridad y salud ocupacional por sus altos costos y consecuentemente la reducción de la competitividad en el mercado.

Sin embargo, los Sistemas de Gestión de Seguridad y Salud Ocupacional implementados en las empresas de todo tipo y tamaño, demuestran fehacientemente ser procesos dinámicos que permiten adoptar una política activa en la prevención, reduciendo los altos costo por siniestralidad laboral y generando rendimiento económicos altos con resultados monetarios rentables. Según estudios de la Agencia Europea para la Seguridad y Salud en el Trabajo el costo de los accidentes de trabajo y las enfermedades profesionales fluctúa entre 2,6 \% y $3,8 \%$ del producto nacional bruto (PIB) en la mayoría de los países.

El trabajo es un derecho y un deber social, y un derecho económico, fuente de realización personal y base de la economía. El Estado garantizará a las personas trabajadoras el pleno respeto a su dignidad, una vida decorosa, remuneraciones y retribuciones justas y el desempeño de un trabajo saludable y libremente escogido o aceptado. (Constitucional, 2008)

Por esta razón los organismos de control interno han desarrollado e implementado normativas técnico - legales en materia de prevención de riesgos laborales apuntado a responsabilizar a la sociedad en la cultura preventiva, a fin de crear conciencia.

A través del presente estudio, la entidad productora de piñas pretende sentar una línea base para prevenir y controlar los riesgos asociados a su actividad laboral de modo eficaz y 


\section{Evaluación ergonómica de los puestos de trabajo del área operativa de siembra y empaque}

Vol. 1, núm. 5., (2017)

Karles Hoffman Jaramillo; Johnny Parraga Bravo; Roberto Idrovo Toledo eficiente, a fin de precautelar al trabajador y su situación de bienestar social y colectivo, logrando además la mejora en los procesos de la organización.

\section{Materiales y Métodos.}

La investigación documental es `aquella que se realiza a través de la consulta de documentos (libros, revistas, periódicos, memorias, anuarios, registros, códices, constituciones, etc.). La de campo o investigación directa es la que se efectúa en el lugar y tiempo en que ocurren los fenómenos objeto de estudio. La investigación mixta es aquella que participa de la naturaleza de la investigación documental y de la investigación de campo. (Zorrilla ,1993:43) (Grajales, 2000)

En un universo de trabajo en donde se desea aplicar un análisis estadístico, cuando el muestreo cubre a todos los elementos de la población., se realiza un censo. En muchos de los casos, la realización de un censo no es posible por ser muy costoso, muy extenso o que la muestra se destruya como resultado del análisis. En tales oportunidades se debe practicar un análisis muestral. La muestra es una parte seleccionada de la población que deberá ser representativa, es decir, reflejar adecuadamente las características que deseamos analizar en el conjunto en estudio. (Hernández Sampieri, Fernández Collado, \& Baptista Lucio, 2003)

Con el fin de llevar a cabo el siguiente estudio se ha realizo una visita a las instalaciones de Agroeden, con objeto de tomar los datos oportunos para la evaluación solicitada. De un total de 245 trabajadores que laboran en la empresa, el estudio se enfocó en los procesos de Siembra y Empaque, donde se trabajó con una muestra para el área de Siembra de 25 trabajadores y para el área de Empaque de 45 trabajadores. 


\section{Evaluación ergonómica de los puestos de trabajo del área operativa de siembra y empaque}

Vol. 1, núm. 5., (2017)

Karles Hoffman Jaramillo; Johnny Parraga Bravo; Roberto Idrovo Toledo

Durante 1 semana se realizó el levantamiento de información para los procesos

mencionados de Siembra y Empaque, para un promedio de 70 trabajadores en los siguientes cargos:

Proceso: Siembra:

Puestos de Trabajo: Abastecedor, Sembrador, Auxiliar de cultivo 1, Auxiliar de cultivo 2

\section{Proceso: Empaque}

Puestos de Trabajo: Colocador, Limpiador, Desinfección, Sanitización, Armado, Empaquetado.

La Evaluación de los riesgos fue realizada tomando en cuenta el análisis de las condiciones de trabajo, a partir de los datos obtenidos en las observaciones realizadas en el propio lugar de trabajo, las conversaciones mantenidas con los empleados y el resultado de las mediciones que se estimaron necesarias.

A partir del análisis y evaluación de los riesgos mencionados se incluyó la descripción de los riesgos y el control operativo, estableciendo una planificación priorizada de las medidas preventivas que se deben adoptar para minimizar los posibles efectos desfavorables.

\section{Resultados.}

Se detectó que existen dos Procesos de mayor riesgo, que son la Siembra y el Empaque, en donde el más crítico es la siembra por la postura que mantiene el trabajador 


\section{Evaluación ergonómica de los puestos de trabajo del área operativa de siembra y empaque}

Vol. 1, núm. 5., (2017)

Karles Hoffman Jaramillo; Johnny Parraga Bravo; Roberto Idrovo Toledo

durante sus 8 horas de trabajo y un espacio limitado que impide el poder tener un mejor confort al momento de laborar.

El proceso de empaque si bien es cierto es menso crítico, pero conlleva también movimientos del tronco que hacen que se encasille dentro de los 60 grados, donde aplicando la tabla de la UEM de la unidad de Ergonomía se observa que la corrección se debería dar hasta un movimiento de 48 grados como máximo, lo cual no es así, y como no se aplican unas pausas activas constantes, se pueden tener complicaciones a largo plazo.

Dentro de los 4 puestos de trabajos más críticos evaluados para los procesos de Siembra y Empaque, se analizó que el puesto de trabajo del Sembrador tiene el puntaje más bajo con el 54\%, a comparación de los otros que llegan hasta un $88 \%$ de aceptabilidad, lo cual da una rápida visión de actuación inmediata ante esta situación que por años se lleva a cabo en la empresa exportadora Agroeden, cabe notar que es el trabajo más duro e incómodo por su postura, falta de pausas, y riesgos físicos por temperaturas anormales, radiaciones no ionizantes.

\section{Conclusiones.}

A lo largo del estudio ergonómico para los 4 puestos de trabajo: Empaquetador, Colocador, Abastecedor y sembrador, se apreció algo muy peculiar, que no solo es la falta de capacitación en la parte ergonómica, sino una forma de trabajo muy enraizada en las personas que laboran en el área agrícola en los diferentes tipos de plantaciones en el Ecuador: como banano, cacao, frutas, legumbres entre otros, es decir que ellos saben y conocen los riesgos pero por la premura de 


\section{Evaluación ergonómica de los puestos de trabajo del área operativa de siembra y empaque}

Vol. 1, núm. 5., (2017)

Karles Hoffman Jaramillo; Johnny Parraga Bravo; Roberto Idrovo Toledo

cumplir en el menor tiempo posible con su tarea y salir temprano, rompen esas normativas poniendo en riesgo su estado físico y la salud, hay que luchar contra esa Idiosincrasia.

\section{Recomendaciones.}

Las propuestas de acción para mitigar los problemas ergonómicos de esta empresa, Empiezan desde la alta Gerencia, participando y motivando a todo el personal en mantener una postura de trabajo acorde a las normativas internacionales, que garantizan un bienestar para su salud.

Para garantizar una revisión y control de los riesgos laborales detectados en el área ergonómica, se desarrolló un "Programa Anual de Control de Actividades Ergonómicas" que consta de las siguientes partes:

Propósito

Alcance

Responsables

Programa

El programa de actividades de control ergonómico permitirá identificar mediante un enfoque técnico los pasos a seguir en el proceso de la mejora en las condiciones de trabajo, sin embargo en el proceso se pueden presentar eventos que no permitan el desarrollo del mismo en 


\section{Evaluación ergonómica de los puestos de trabajo del área operativa de siembra y empaque}

Vol. 1, núm. 5., (2017)

Karles Hoffman Jaramillo; Johnny Parraga Bravo; Roberto Idrovo Toledo

su totalidad, para ello es importante tomar acciones en estos casos y re-planificar el programa a fin de que se obtengan los resultados esperados.

Se debe considerar a este programa como un panorama general que abarcara todos los correctivos, el enfoque que se debe tomar para que se generen buenos resultados será la objetividad del mismo.

El compromiso de la mejora continua dentro de la empresa procesadoras de Piña AGROEDEN, dará como resultado un buen sistema de gestión en seguridad y salud.

\section{Bibliografía}

Constitucional, T. (2008). Constitución de la República del Ecuador. Quito: Registro Oficial.

Grajales, T. (27 de 03 de 2000). Recuperado el 10 de 12 de 2017, de http://tgrajales.net/investipos.pdf

Hernández Sampieri, R., Fernández Collado, C., \& Baptista Lucio, P. (2003). Metodología de la investigación (Vol. 707). Mexico: McGraw-Hill.

Parra, M. (2003). Conceptos básicos en salud laboral. Santiago de Chile.

Polanco Trochez, E., Rodríguez Mosquera, D., \& Vidal Mosquera, M. (22 de 09 de 2011). Universidad Catolica de Manizales. Recuperado el 10 de 12 de 2017, de Biblioteca Hna. Josefina Nuñez Gomez: http://hdl.handle.net/10839/142

Rodríguez, M. (2009). Rodríguez, M. (2009). Factores psicosociales de riesgo laboral: ¿ nиevos tiempos, nuеvos riesgos?

Salinas Garrido, R. (2008). Seguridad y Medio Ambiente. Recuperado el 10 de 12 de 2017, de http://www.seguridadypromociondelasalud.com/n109/articulo1.html 